\title{
RENTA PER CAPITA MUNICIPAL EN SEVILLA (1977-1988)
}

\author{
José Pérez Blanco \\ Catedrático de Teoria Económica \\ Universidad de Sevilla
}

El presente estudio, tiene por objeto dar a conocer las investigaciones del autor (1), en torno a la estimación de los ingresos personales a nivel municipal, en todos y cada uno de los ciento dos municipios sevillanos.

Se actualiza asi, el ya publicado anteriormente, sobre el mismo tema pero con un modelo matemático diferente.

Estimamos que este trabajo tiene interés generalizado para los municipios pues posibilita el cálculo estimativo de dicha magnitud económica con suma facilidad y por esa razón, se trae a las páginas de esta Revista.

\section{1.- El modelo matemático.}

El sistema se basa en estimar la renta personal disponible a través de un método indirecto, lo que, antiguamente y por similitud con ya olvidados procedimientos fiscales, podría denominarse "signos externos".

Ya en 1969-71, el método se utilizaba en el Ayuntamiento de Barcelona y anteriormente (primeros años de la década de los sesenta) en paises como Alemania, Francia y Noruega, como una aplicación del análisis de renta o de riqueza.

Muchos pudieran ser tales índices, pero -lo importante- es que tengan significado actual, $y$ denoten $e$ indicien la magnitud que, pretendemos, averiguar. Se han utilizado a tal fin, el número de vehículosturismos por cada 1000 habitantes, el de teléfonos por cien, diversos electrodomésticos por tantos hogares, etc. El autor, utilizó el indicador mixto motorización-electrificación desde 1960 a 1977, y desde 1977 a 1986, sólo el de consumo de electricidad para usos domésticos, expresados en kw. por habitante y año.

En el estudio anteriormente publicado, el modelo venía dado por la expresión:

$y=0,405 x+111,78$

donde la variable "X" se expresa en kxh/año para cada municipio, resultante el valor de "y", muy asimilable a renta per cápita disponible,

(1).- Este trabajo ha contado con la eficaz colaboración de la catedrática de Estadística, Dña. Dolores Valenzuela y García de Polavieja. 
expresada en miles de Ptas. de valor constante de 1980.

Los datos de consumo, los ha venido y viene facilitando, amablemente, Sevillana de electricidad, S.A.., en un expreso deseo de colaboración con esta línea -y otras muy diversas- de la investigación universitaria.

Era deseo del autor, ratificar la significación que siguiera teniendo el indicador elegido en el cálculo de la renta personal y por ello, por encargo de la Diputación de Sevilla (2), elaboró un nuevo modelo basado en la correlación calculada entre dos variables: renta per cápita a nivel nacional y consumo por habitante de electricidad para usos domésticos, en una amplia serie que abarca los años 1961-1988.

La bondad del ajuste se pone de relieve al considerar que el coeficiente de significación es 0,9842 , lo que indica -aproximadamente- que la variable "electrificación doméstica", explica -por sí sola- el $98,42 \%$ de las cifras de renta media personal (casi la indica totalmente).

En el cuadro $A$, en el anexo, se especifican los datos que han servido de base para la construcción de la recta de regresión.

El gráfico insertado refleja la bondad del ajuste estadístico de nuestro modelo.

Mediante los oportunos cálculos, dicho modelo construído fue:

$y=0,3829 x+112,987$

con la misma significación dada, anteriormente, a las variables.

Como se observará, ambos modelos difieren escasamente, e incluso el coeficiente de correlación entre ambos, es bastante similar.

Se confirma asi la persistencia del consumo de energía eléctrica en el hogar como indicador de nivel de vida. Efectivamente, tal índice, engloba no sólo el consumo para alumbrado, sino lo más importante, el uso de aparatos electrodomésticos, muy progresivo en nuestro país.

\section{2.- La serie temporal en los municipios sevillanos.}

Conocidos los datos del consumo de electricidad por habitante $y$ año, se construyó la tabla núm. 1 («Renta per cápita: Municipios de Sevillan), que la expresa en orden alfabético, e incluye -al final- la media provincial y la nacional para cada uno de los años, así como el porcentaje que la media provincial representa sobre aquella.

Es preciso advertir que las medias supramunicipales se han obtenido aplicando la misma recta de regresión a las cifras de consumos medios, provincial y nacional, de electricidad, a fin de hacer más homogéneos los

(2).- El trabajo ha sido editado recientemente por la Diputación de Sevilla, bajo el título "Estimaciones de los ingresos personales en los municipios de la provincia de Sevilla (1985-88). Biblioteca de Socieconomía Sevillana. Núm. 3. Págs. 179. 1000 ej. año 1990. 
términos de la comparación y aunque dichas medias pueden ser conocidas por otros trabajos (3).

De la comparación aludida, se desprenden dos características de la provincia de Sevilla, en su conjunto:

a) Es una provincia subdesarrollada con respecto a la media nacional.

En efecto, la renta media provincial, en el mejor de los casos (año 1986 ) se queda en el $92,47 \%$ de la nacional y en el peor (1980), en tan sólo el $78,36 \%$.

b) Una nota de optimismo, la pone el hecho de que, pese a dicho retraso relativo, se acorta camino -en estos últimos 12 años- en la distancia que separa la provincia de la media de España. Del 80,54\% en 1977 , se pasa al $89,57 \%$ en 1988.

Pero, lo que más evidencia el mayor ritmo de crecimiento de la provincia de Sevilla, es la comparación del indice simple de incremento entre 1977-88. El indice medio provincial es $139,41(39,41 \%$ de aumento de la renta) frente a 125,35 a nivel nacional medio (sólo el 25,35\%).

No pretendemos, en este apretado artículo, exponer los muy variados análisis y conclusiones que se han obtenido en la investigación (4). No obstante, parece interesante señalar las tasas medias anuales acumulativas de incremento de la renta per cápita provincial $(2,99 \%)$, y nacional $(2,08 \%)$, cuya diferencia indica, por sí misma, el despegue económico de la provincia de Sevilla.

También en dicha tabla, podrá observarse el diferente ritmo de crecimiento entre los municipios, encuadrados en diferentes Comarcas, según el número que lo indica en la primera columna y que las distingue: (1).- Sierra Norte.- (2).- Valle del Guadalquivir.- (3).- Campiña-Marismas.(4),- Zona de influencia de la Capital.- (5) .- El Aljarafe.- (6).- Sierra Sur.

3.- Diferencia de la tasa de crecimiento municipal.

En la siguiente tabla núm. 2. (Indice de crecimiento: orden descendente), se ha versionado la anterior, pero ordenando a los 102 municipios según el índice de aumento de la renta per cápita entre 197788 y que figura en la última columna.

La dinamicidad en el desarrollo es muy diversa, pero destacan las siguientes notas:

a) Tan sólo cinco municipios sevillanos crecieron menos que la media del país. fueron éstos: Almadén de la Plata, Castilleja de la Cuesta, San Nicolás del Puerto, El Garrobo y Aznalcóllar. Todos ellos, salvo

(3).-Existen cálculos de la renta a nivel nacional y provincial en las conocidas publicaciones del Banco de Bilbao (Renta Nacional y su distribución provincial) y de Banesto (Anuarios del Mercado Español).

(4).- :Las Haciendas Municipales en la Provincia de Sevilla» (1974-80-. Edit. Instituto de Desarrollo Regional de la Universidad de Sevilla. Año 1985. 1000 ej. 
Castilleja, enclavados en la pobre Comarca de la Sierra Norte.

Anómalo es el caso de Castilleja de la Cuesta, municipio dormitorio de Sevilla y aunque hemos contrastado minuciosamente la información facilitada, sólo puede explicarse el bajo nivel de crecimiento por el escaso término municipal que tiene y que impide una mayor expansión urbana o de segundas residencias, ya que dicho municipio está enclavado en el Aljarafe, zona pródiga en urbanizaciones de recreo y con un gran crecimiento demográfico.

Incluso, sólo 53 municipios crecieron menos (respecto a renta personal) que la propia media provincial. Aproximadamente pues, un $50 \%$ de ellos quedan por encima y el otro $50 \%$ por debajo de dicho promedio.

Por el número de Comarca al que se adscribe cada municipio y observando los que quedan por encima de la media provincial, se puede constatar que no existe ninguna identificación exacta entre mayor por ciento de crecimiento y ubicación de los municipios en una determinada Comarca.

A dicho respecto, la observación más importante y a la vez sorprendente, es que -precisamente- la Comarca más desarrollada históricamente (la Zona de Influencia de Capital), es la que cuenta con menor número de pueblos entre los que se sitúan por encima del promedio provincial, como si fuera lo más difícil forzar el ritmo de desarrollo, mientras más rico es el municipio o la comarca de que se trate.

\section{4.- La clasificación comarcal.}

En la tabla núm. 3, los datos se versionan, agrupando los municipios por Comarcas y dentro de cada una de ellas, siguen un orden decreciente respecto al índice de crecimiento.

Al final de los datos municipales de cada Comarca, se introducen comparaciones entre los promedios comarcal, provincial y nacional.

Las Comarcas definidas, lo fueron ya en la Tesis Doctoral del autor (4), en 1980, en base a la observación empírica y el conocimiento de la provincia que tiene el autor, como Economista que fue de la Diputación de Sevilla entre 1971-82. Dicha observación fue ampliamente corroborada por los presentes estudios de rentas y el grado de homogeneidad y cohesión que se observa, con independencia del análisis espacial y factorial que se está realizando en la actualidad, al respecto.

Estas son las caracteristicas de las seis Comarcas definidas:

1.- Sierra Norte. (Número 1). Comprende 20 municipios enclavados en Sierra Morena y sus estribaciones. Se caracteriza por una agricultura pobre, malas comunicaciones, escaso nivel de 
servicios y muy baja industrialización. De ahi que sea de las Comarcas más marginadas de la provincia, pese a las cuantiosas inversiones que se vienen realizando desde 1971, al menos. Habitan aquí el 4,32\% de la población provincial, que se reparten el $3,15 \%$ de la renta.

2.- Sierra Sur (núm.6), con muy similares características a la anterior, pero mejores comunicaciones y mayor productividad agraria. Población: $6,74 \%$. Renta: 4,86\%.

3.- Valle del Guadalquivir. (núm.2). Agricultura de regadío; comunicaciones aceptables; nivel intermedio del sector servicios e incipiente industrialización. Población: 6,42\%. Renta: 5,04\%.

4.- Campiña-Marismas. (núm. 3). Buenas comunicaciones; agricultura de secano, con alta productividad; industrialización incipiente. Población: 19,07\%. Renta: 15,97\%.

5.- Aljarafe (núm. 5). Buena distribución de la tierra; agricultura de secano; olivar; girasol y cereales. Comunicaciones excelentes; desarrollo de los servicios e industrialización creciente. Zona de creciente urbanización con enorme actividad constructora. Población: 7,91\%. Renta: 7,72\%.

6.- Zona de Influencia de la Capital. Compuesta por Sevilla y municipios dormitorios, es la zona más desarrollada de la provincia, tanto por ser la de mayor industrialización y de servicios desarrollados. Agricultura prácticamente nula. Población: 55,54\%. Renta: $62,22 \%$.

El orden en que se han expuesto las diversas Comarcas no ha sido caprichoso. Como se observará fácilmente, en todas las Zonas, salvo en la última, es más alto el porcentaje de población que el de la renta total de la misma, lo que significa, por un lado, menor desarrollo en las primeramente enumeradas $y$, en segundo lugar, una alta concentración de la población en una sola de las Comarcas: la de la Capital.

Por otro lado, la equidistribución de la renta-dentro de cada Comarcay entre los diversos municipios es bastante aceptable, como lo delatan los diversos índices de Gini, que -en el peor de los casos- no excede de 0,25 . Este argumento incide en la aseveración del acierto en la división Comarcal que venimos utilizando.

\section{5.- Conclusiones.}

Importa muy mucho, y ese es el objetivo esencial del presente artículo, poner de manifiesto que el procedimiento utilizado, con las modificaciones $y$ mejoras que se pudieran introducir, constituye un método fácil y poco costoso a fin de aproximar un concepto de renta personal media disponible para espacios menores que la provincia, e 
incluso aplicable a distritos municipales de las capitales de provincia (5).

Evidentemente, lo más importante de este trabajo no es la exposición concreta de las series que aparecen en los cuadros que se insertan, pues su interés pudiera quedar reducido a los habitantes de la provincia de Sevilla, sino la posibilidad de dar a conocer la metodología para que pueda generalizarse a otros espacios territoriales.

Estos datos tienen muy diversas aplicaciones, en especial cuando se trata de valorar la situación de los diferentes municipios a efecto de políticas económicas de creación de infraestructuras, ayudas y subvenciones a las zonas, comarcas o municipios más deprimidos.

Ig 'almente, pudiera servir a los rectores y municipales para graduar la carga fiscal de su competencia y para fines similares a otros niveles.

Respecto a los datos de la provincia de Sevilla, se puede poner de manifiesto, esencialmente, el mayor dinamismo de crecimiento de la misma, por encima de la media nacional, lo que le ha permitido, entre 1977-88, acortar distancias y aproximarse a un mayor nivel de vida, similar, al menos, al promedio de este país.

(5).- "Los ingresos personales en los distritos municipales de Sevilla" (año 1986-88). Edit. Excmo. Ayuntamiento de Sevilla. Año 1989. 1000 ej. 


\section{CUADRO A}

\section{DATOS BÁSICOS PARA CALCULAR EL MODELO}

\begin{tabular}{|c|c|c|c|}
\hline AÑOS & $\begin{array}{l}\text { KWH/HB } \\
\text { U. DOM. } \\
\text { MD NC (1) }\end{array}$ & RPC (2) MD NAC. & $\begin{array}{c}\text { RPC (2) VALORES } \\
\text { AJUSTADOS MOD. } \\
1988\end{array}$ \\
\hline 1961 & 174,94 & 169,600 & 179,978 \\
\hline 1962 & 194,11 & 185,914 & 187,320 \\
\hline 1963 & 217,17 & 196,124 & 196,149 \\
\hline 1964 & 247,14 & 204,541 & 207,628 \\
\hline 1965 & 255,59 & 215,181 & 210,865 \\
\hline 1966 & 288,53 & 230,080 & 223,277 \\
\hline 1967 & 318,32 & 239,489 & 234,883 \\
\hline 1968 & 355,35 & 251,756 & 249,065 \\
\hline 1969 & 398,75 & 268,689 & 265,684 \\
\hline 1970 & 434,48 & 280,443 & 279,369 \\
\hline 1971 & 462,08 & 292,354 & 289,938 \\
\hline 1972 & 507,93 & 316,020 & 307,493 \\
\hline 1973 & 612,86 & 341,805 & 347,678 \\
\hline 1974 & 608,57 & 356,421 & 346,034 \\
\hline 1975 & 624,15 & 354,937 & 352,001 \\
\hline 1976 & 656,77 & 361,174 & 364,492 \\
\hline 1977 & 676,18 & 363,280 & 371,923 \\
\hline 1978 & 671,82 & 366,898 & 370,257 \\
\hline 1979 & 675,60 & 360,680 & 371,701 \\
\hline 1980 & 651,51 & 359,773 & 362,479 \\
\hline 1981 & 654,12 & 350,850 & 363,477 \\
\hline 1982 & 652,79 & 351,743 & 362,967 \\
\hline 1983 & 641,87 & 354,478 & 358,786 \\
\hline 1984 & 667,78 & 360,117 & 368,708 \\
\hline 1985 & 685,49 & 367,311 & 375,489 \\
\hline 1986 & 697,94 & 382,471 & 380,258 \\
\hline 1987 & 714,86 & 404,056 & 386,737 \\
\hline 1988 & 733,21 & 422,479 & 393,764 \\
\hline
\end{tabular}

(1).- KWH/HABIT/AÑO (MEDIA NACIONAL): (X)

(2).- RENTA PER CAP. MILES DE PTAS DE 1980. MEDIA SEGÚN SERVICIO ESTUDIOS DEL BBV

(3).- RENTA PER CÁPITA (ID. ID.) EN VALORES AJUSTADOS 
TABLA NÚM. 1.- RENTA PER CÁPITA: MUNICIPIOS DE SEVILLA

RENTA PER CÁPITA (PTAS DE 1980)

\begin{tabular}{|c|c|c|c|c|c|c|c|c|c|}
\hline $\begin{array}{l}\text { NOM. } \\
\text { COMARC }\end{array}$ & MENICIIOS & 1977 & 1980 & 1982 & 1984 & 1986 & 1987 & 1988 & $\begin{array}{l}\text { CNDICR } \\
\text { INCRMTO } \\
1988.87\end{array}$ \\
\hline 6 & Aguadulce & 171172 & 181004 & 193941 & 197242 & 237938 & 248583 & 261098 & 152,54 \\
\hline 1 & Alanis & & 981 & 246 & 195206 & 77681 & 3882 & 223658 & 135,92 \\
\hline 5 & Albaida & & 204686 & 907 & 234075 & 227186 & 240283 & 247049 & 127,67 \\
\hline 4 & $\mathrm{Alc}$ & 4845 & 269698 & 296347 & 305877 & 328370 & 3775 & 362233 & 142,14 \\
\hline 2 & Alcalá & 0211 & 211786 & 227937 & 252494 & 260118 & 257656 & 296362 & 148,02 \\
\hline 2 & Alcole & 1481 & 202441 & 211311 & 219024 & 244633 & 246924 & 245875 & 128,41 \\
\hline 2 & & 1791 & 202283 & 220517 & 226560 & 246099 & 247077 & 271628 & 149,42 \\
\hline 6 & & 67919 & 166957 & 240234 & 225354 & 207247 & 211605 & 216164 & 136,88 \\
\hline 1 & Almadén d & 166997 & 176579 & 222223 & 233132 & 192049 & 196269 & 200713 & 120,19 \\
\hline 5 & & 0118 & 201087 & 182617 & 257204 & 274714 & 275974 & 331683 & 174,46 \\
\hline 3 & & 3937 & 183935 & 206322 & 225688 & 238836 & 244077 & 257769 & 148,20 \\
\hline 5 & & 137 & 197927 & 219699 & 235682 & 242939 & 251366 & 258108 & 137,92 \\
\hline 1 & & 475 & 197226 & 244965 & 235759 & 184671 & 187797 & 191059 & 102,46 \\
\hline 6 & & 9890 & 169046 & 179780 & 193751 & 201007 & 217741 & 228751 & 143,07 \\
\hline 5 & & & 20109 & 212126 & 233714 & 26004 & 292019 & 331006 & 184,14 \\
\hline 5 & & & 4592 & & 231795 & 248290 & 267075 & 288469 & 149,14 \\
\hline 5 & & 210910 & 223126 & 242360 & 262604 & 270480 & 278771 & 298585 & 141,57 \\
\hline 2 & & 98498 & 209971 & 234622 & 249699 & 254511 & 260812 & 303355 & 152,83 \\
\hline 1 & & 1343 & 191786 & 212995 & 213166 & 239548 & 242240 & 249760 & 137,73 \\
\hline 3 & Cabezas & 4108 & 194717 & 224470 & 37981 & 254081 & 259779 & 272201 & 147,85 \\
\hline 4 & & 0023 & 243386 & 253895 & 288032 & 09027 & 3118112 & 334542 & 145,44 \\
\hline 3 & & & 9235 & 220061 & 232104 & 245501 & 260406 & 276987 & 140,03 \\
\hline 1 & & & & & 244759 & & & 289220 & 144,95 \\
\hline 3 & & 7944 & 230584 & 253901 & 304537 & 322134 & 328324 & 378168 & 173,52 \\
\hline 5 & Carrión & 182121 & 192610 & 214470 & 225956 & 226662 & 237343 & 247087 & 135,67 \\
\hline 6 & & 166259 & 175797 & 195045 & 210255 & 209419 & 215542 & 237101 & 142,61 \\
\hline 1 & Cassiblan & 14271 & 191876 & 220785 & 47516 & 256763 & 245941 & 291066 & 160,43 \\
\hline 5 & & 2716 & 214441 & 222872 & 246597 & 52912 & 290277 & 322637 & 159,16 \\
\hline 5 & & & 032 & & 351907 & 8097 & 366766 & 376109 & 118,14 \\
\hline 5 & & 205 & 217792 & & 253375 & 267406 & 272258 & 277261 & 134,67 \\
\hline 1 & & 2120 & 224377 & 248 & 270608 & 309346 & 316012 & 322677 & 152,14 \\
\hline 1 & & 1702 & 180009 & 199245 & 215502 & 231459 & 240483 & 261347 & 153,52 \\
\hline 1 & & 167143 & 176734 & 223268 & 244874 & 264369 & 262872 & 278203 & 166,45 \\
\hline 4 & Coria de & 219423 & 232151 & 270316 & 293661 & 317375 & 316881 & 321678 & 146,60 \\
\hline 6 & & 141 & 149687 & 167403 & 180462 & 190571 & 199342 & 220932 & 155,99 \\
\hline 6 & & 171 & 181779 & 211692 & 226608 & 246477 & 253591 & 262614 & 152,77 \\
\hline 6 & Corrales, Los & 143994 & & $1 / 0109$ & 184400 & |1945!! & 201180 & | 217486 & 151,04 \\
\hline
\end{tabular}


TABLA NÚM. 1.- RENTA PER CÁPITA: MUNICIPIOS DE SEVILLA

RENTA PER CÁPITA (PTAS DE 1980)

\begin{tabular}{|c|c|c|c|c|c|c|c|c|c|}
\hline $\begin{array}{l}\text { NÓM. } \\
\text { COMARC }\end{array}$ & MUNCIPIOS & 1977 & 1980 & 1982 & 1984 & 1986 & 1987 & 1988 & \begin{tabular}{|l} 
ADDICE \\
A'CRMTO \\
1988.87
\end{tabular} \\
\hline 4 & Dos Hermanas & 229931 & 243290 & 253023 & 273824 & 274667 & 281952 & 294972 & 128,29 \\
\hline 3 & Ecija & 197982 & 209423 & 240715 & 261264 & 291667 & 296717 & 316176 & 159,70 \\
\hline 5 & Espartinas & 288242 & 305099 & 355575 & 358724 & 404198 & 407235 & 408493 & 141,72 \\
\hline 6 & Estepa & 197657 & 209079 & 222274 & 244606 & 260479 & 265047 & 276147 & 139,71 \\
\hline 3 & Fuentes Andalucía & 176736 & 186902 & 176785 & 187836 & 219316 & 226374 & 250942 & 141,99 \\
\hline 1 & Garrobo, El & 262577 & 220177 & 259766 & 267688 & 276038 & 284838 & 294114 & 112,01 \\
\hline 4 & Gelves & 227645 & 240865 & 270904 & 277960 & 283219 & 288413 & 324769 & 142,66 \\
\hline 1 & Gerena & 184379 & 195545 & 218475 & 235070 & 238343 & 247994 & 277494 & 150,50 \\
\hline 6 & Gilena & 150544 & 159139 & 168772 & 187655 & 279824 & 279833 & 279842 & 185,89 \\
\hline 5 & Gines & 310281 & 328460 & 387276 & 399440 & 411604 & 419964 & 428323 & 138,04 \\
\hline 1 & Guadalcanal & 190507 & 201500 & 216752 & 225459 & 224387 & 232994 & 274383 & 144,03 \\
\hline 2 & Guillena & 175836 & 185949 & 208421 & 225879 & 242677 & 247545 & 274081 & 155,87 \\
\hline 6 & Herrera & 184648 & 195307 & 212217 & 243112 & 258280 & 260814 & 289882 & 156,99 \\
\hline 5 & Huevar & 178910 & 189207 & 201113 & 214878 & 230793 & 249193 & 270468 & 151,18 \\
\hline 3 & Lantejuela, La & 179466 & 189797 & 222602 & 235262 & 239464 & 244758 & 263049 & 147,92 \\
\hline 3 & Lebrija & 177832 & 188063 & 209839 & 228829 & 239464 & 244758 & 263049 & 147,92 \\
\hline 6 & Lora de Estepa & 156689 & 165653 & 183568 & 203056 & 228736 & 253114 & 266441 & 170,04 \\
\hline 3 & Luisiana, La & 149390 & 157916 & 196533 & 208494 & 223194 & 229064 & 246660 & 165,11 \\
\hline 1 & Madroño, El & 171298 & 181138 & 208353 & 222663 & 243123 & 257674 & 272127 & 158,86 \\
\hline 3 & Mairena del Alcor & 221051 & 233876 & 277298 & 316178 & 325682 & 333672 & 430136 & 194,59 \\
\hline 5 & Mairena del Aljarafe & 270589 & 286386 & 303767 & 322889 & 343929 & 367078 & 382521 & 141,37 \\
\hline 3 & Marchena & 193998 & 205190 & 264384 & 284547 & 300174 & 309261 & 335432 & 172,90 \\
\hline 6 & Marinaleda & 152135 & 160826 & 178024 & 187661 & 201419 & 28735 & 219539 & 144,31 \\
\hline 6 & Martín de la Jara & 146962 & 155343 & 171912 & 193482 & 191068 & 202099 & 215520 & 146.65 \\
\hline 3 & Molares, Los & 153381 & 162146 & 187604 & 197548 & 208818 & 221589 & 236063 & 153,91 \\
\hline 6 & Montellano & 161647 & 171409 & 189627 & 205047 & 203556 & 218150 & 233041 & 144,17 \\
\hline 6 & Morón Fronterạ & 202282 & 213982 & 240768 & 252097 & 263427 & 272057. & 289285 & 143,01 \\
\hline 1 & Navas Concepción & 158770 & 167859 & 187964 & 197929 & 192454 & 200398 & 247720 & 156,02 \\
\hline 5 & Olivares & 201341 & 212983 & 239714 & 255099 & 258551 & 266093 & 280798 & 139,46 \\
\hline 3 & Osuna & 178459 & 188729 & 230692 & 245372 & 274439 & 277320 & 281160 & 157,55 \\
\hline 3 & Palacios, Los & 186516 & 197270 & 222108 & 232199 & 252022 & 259562 & 270515 & 145,04 \\
\hline 5 & Palomares del Río & 273748 & 289735 & 307312 & 326637 & 347884 & 371244 & 396928 & 145,00 \\
\hline 3 & Paradas & 168886 & 178581 & 197401 & 210179 & 236714 & 232952 & 251801 & 149,10 \\
\hline 6 & Pedrera & 170620 & 180420 & 201441 & 209605 & 226670 & 237022 & 232891 & 136,50 \\
\hline 1 & Pedroso, El & 180263 & 190641 & 210198 & 216689 & 230208 & 240148 & 267710 & 148,51 \\
\hline 2 & Peñaflor & 176184 & 186318 & 206504 & 220059 & 225916 & 226736 & 255036 & 144,76 \\
\hline 5 & Pilas & 210870 & 223084 & 230878 & 254443 & 263794 & 274445 & 300042 & 142,29 \\
\hline
\end{tabular}


TABLA NÚM. 1.- RENTA PER CÁPITA: MUNICIPIOS DE SEVILLA RENTA PER CÁPITA (PTAS DE 1980)

\begin{tabular}{|c|c|c|c|c|c|c|c|c|c|}
\hline $\begin{array}{l}\text { NÚM. } \\
\text { COMARC }\end{array}$ & MUNICIPIOS & 1977 & 1980 & 1982 & 1984 & 1986 & 1987 & 1988 & $\begin{array}{l}\text { ÍNDICE } \\
\text { INCRMTO } \\
1988.87\end{array}$ \\
\hline 6 & Pruma & 166618 & 176178 & 187441 & 200712 & 216349 & 234772 & 256480 & 153,93 \\
\hline 3 & Puebla de Cazalla & 179003 & 179882 & 208276 & 228522 & 241080 & 246721 & 279453 & 156,09 \\
\hline 1 & Puebla Infantes & 157551 & 166566 & 189402 & 200644 & 211282 & 216582 & 229579 & 145,72 \\
\hline 4 & Puebla del Río & 224474 & 237505 & 266693 & 285505 & 323066 & 323066 & 324197 & 331675 \\
\hline 1 & Real de la Jara & 155839 & 164752 & 159476 & 164810 & 196443 & 193724 & 199254 & 127,86 \\
\hline 2 & Rinconada,La & 216543 & 229098 & 251129 & 263178 & 275411 & 289899 & 301193 & 139,09 \\
\hline 6 & Roda de Andalucía & 187761 & 198589 & 214169 & 229231 & 232242 & 237999 & 260230 & 138,60 \\
\hline 1 & Ronquillo, El & 193574 & 204750 & 214916 & 221667 & 250945 & 257674 & 298680 & 154,30 \\
\hline 6 & Rubio, El & 174587 & 184625 & 197642 & 209069 & 222705 & 233385 & 240252 & 137,61 \\
\hline 5 & Salteras & 213947 & 226346 & 248973 & 256705 & 277285 & 291402 & 305913 & 142,99 \\
\hline 4 & S. Juan Aznalfarache & 275494 & 291586 & 320543 & 338542 & 406216 & 396417 & 386944 & 140,45 \\
\hline 5 & Sanlucar la Mayor & 262814 & 278145 & 313306 & 321310 & 331676 & 338958 & 346483 & 131,84 \\
\hline 1 & San Nicolfs Puerto & 184713 & 188966 & 193472 & 198244 & 203300 & 208656 & 214329 & 116,03 \\
\hline 4 & Santiponce & 213223 & 225579 & 255595 & 274743 & 270850 & 273676 & 302342 & 141,80 \\
\hline 6 & Saucejo, El & 148317 & 156779 & 170714 & 180998 & 188793 & 200608 & 209648 & 141,35 \\
\hline 4 & Sevilla & 326174 & 345307 & 334699 & 365348 & 408018 & 408540 & 432501 & 132,60 \\
\hline 2 & Tocina & 193992 & 205194 & 264566 & 253950 & 273953 & 278982 & 283677 & 146,23 \\
\hline 5 & Tomares & 266969 & 282550 & 329590 & 348614 & 351276 & 381155 & 411033 & 153,96 \\
\hline 5 & Umbrete & 200341 & 211924 & 253401 & 263715 & 281178 & 291831 & 317752 & 158,61 \\
\hline 3 & Utrera & 200616 & 212215 & 234217 & 253153 & 268992 & 276785 & 306943 & 153,00 \\
\hline 5 & Valencina Concep. & 302584 & 320302 & 338853 & 363549 & 438511 & 468571 & 495429 & 163,73 \\
\hline 5 & Villamanrique Cond. & 176830 & 187002 & 179670 & 187445 & 251163 & 256481 & 250725 & 141,79 \\
\hline 5 & Villanueva Ariscal & 251667 & 266330 & 300871 & 332645 & 344074 & 355503 & 378009 & 150,20 \\
\hline 1 & Villan. Río y Minas & 178839 & 189131 & 216954 & 224147 & 275579 & 281213 & 287042 & 160,50 \\
\hline 6 & Villan. de San Juan & 129289 & 136609 & 159972 & 164758 & 198183 & 206720 & 207861 & 160,77 \\
\hline 2 & Villaverde del Río & 194444 & 205673 & 217224 & 228254 & 253398 & 258887 & 265601 & 136,60 \\
\hline 3 & Viso del Alcor, El & 188213 & 199068 & 233853 & 251498 & 255528 & 266576 & 306322 & 162,75 \\
\hline & Media nacional & 319012 & 349265 & 349743 & 362058 & 359444 & 378291 & 399894 & 125,35 \\
\hline & Media provincial & 196166 & 206885 & 228918 & 244632 & 260767 & 268796 & 287414 & 146,52 \\
\hline & $\%$ md. prov. s/nac. & 61,49 & 59,23 & 65,45 & 67,57 & 72,55 & 71,06 & 71,87 & \\
\hline
\end{tabular}


TABLA NÚM. 2.-CLASTFICACIÓN POR ORDEN DECRECLENTE DEL ÍNDICE DE AUMENTO DE LA RENTA PERSONAL

\begin{tabular}{|c|c|c|c|c|c|c|c|c|c|}
\hline \multicolumn{10}{|c|}{ RENTA PER CÁPITA (PTAS DE 1980) } \\
\hline $\begin{array}{c}\text { N'M. } \\
\text { COMARC }\end{array}$ & MLNICIPIOS & 1977 & 1980 & 1982 & 1984 & 1986 & 1987 & 1988 & $\begin{array}{l}\text { ĹDICE } \\
\text { RCCRNTO } \\
1988.87\end{array}$ \\
\hline 3 & Mairena del Alcor & 221051 & 233876 & 277298 & 316178 & 325682 & 333672 & 430136 & 194,59 \\
\hline 6 & Gilena & 150544 & 159139 & 168772 & 187655 & 279824 & 279833 & 279842 & 185,89 \\
\hline 5 & Benacazín & 179761 & 190109 & 212126 & 233714 & 26004 & 292019 & 331006 & 184,14 \\
\hline 5 & Almensilla & 190118 & 201087 & 182617 & 257204 & 274714 & 275974 & 331683 & 174,46 \\
\hline 3 & Carmona & 217944 & 230584 & 253901 & 304537 & 322134 & 328324 & 378168 & 173,52 \\
\hline 3 & Marchena & 193998 & 205190 & 264384 & 284547 & 300174 & 309261 & 335432 & 172,90 \\
\hline 6 & Lora de Estepa & 156689 & 165653 & 183568 & 203056 & 228736 & 253114 & 266441 & 170,04 \\
\hline 1 & Constantina & 167143 & 176734 & 223268 & 244874 & 264369 & 262872 & 278203 & 166,45 \\
\hline 3 & Luisiana, La & 149390 & 157916 & 196533 & 208494 & 223194 & 229064 & 246660 & 165,11 \\
\hline 5 & Valencina Concep. & 302584 & 320302 & 338853 & 363549 & 438511 & 468571 & 495429 & 163,73 \\
\hline 3 & Viso del Alcor, El & 188213 & 199068 & 233853 & 251498 & 255528 & 266576 & 306322 & 162,75 \\
\hline 6 & Villan. de San Juan & 129289 & 136609 & 159972 & 164758 & 198183 & 206720 & 207861 & 160,77 \\
\hline 1 & Villan. Río y Minas & 178839 & 189131 & 216954 & 224147 & 275579 & 281213 & 287042 & 160,50 \\
\hline 1 & Castilblanco Arroyos & 1814271 & 191876 & 220785 & 247516 & 256763 & 245941 & 291066 & 160,43 \\
\hline 3 & Ecija & 197982 & 209423 & 240715 & 261264 & 291667 & 296717 & 316176 & 159,70 \\
\hline 5 & Castilleja del Campo & 202716 & 214441 & 222872 & 246597 & 262912 & 290277 & 322637 & 159,16 \\
\hline 1 & Madroño, El & 171298 & 181138 & 208353 & 222663 & 243123 & 257674 & 272127 & 158,86 \\
\hline 5 & Umbrete & 200341 & 211924 & 253401 & 263715 & 281178 & 291831 & 317752 & 158,61 \\
\hline 3 & Osuna & 178459 & 188729 & 230692 & 245372 & 274439 & 277320 & 281160 & 157,55 \\
\hline 6 & Herrera & 184648 & 195307 & 212217 & 243112 & 258280 & 260814 & 289882 & 156,99 \\
\hline 3 & Puebla de Cazalla & 179003 & 179882 & 208276 & 228522 & 241080 & 246721 & 279453 & 156,09 \\
\hline 1 & Navas Concepción & 158770 & 167859 & 187964 & 197929 & 192454 & 200398 & 247720 & 156,02 \\
\hline 6 & Coripe & 141628 & 149687 & 167403 & 180462 & 190571 & 199342 & 220932 & 155,99 \\
\hline 2 & Guillena & 175836 & 185949 & 208421 & 225879 & 242677 & 247545 & 274081 & 155,87 \\
\hline 1 & Ronquillo, El & 193574 & 204750 & 214916 & 221667 & 250945 & 257674 & 298680 & 154,30 \\
\hline 5 & Tomares & 266969 & 282550 & 329590 & 348614 & 351276 & 381155 & 411033 & 153,96 \\
\hline 6 & Pruma & 166618 & 176178 & 187441 & 200712 & 216349 & 234772 & 256480 & 153,93 \\
\hline 3 & Molares, Los & 153381 & 162146 & 187604 & 197548 & 208818 & 221589 & 236063 & 153,91 \\
\hline 1 & Calzada de la Sierra & 170233 & 180009 & 199245 & 215502 & 231459 & 240483 & 261347 & 153,52 \\
\hline 3 & Utrera & 200616 & 212215 & 234217 & 253153 & 268992 & 276785 & 306943 & 153,00 \\
\hline 2 & Brenes & 198498 & 209971 & 234622 & 249699 & 254511 & 260812 & 303355 & 152,83 \\
\hline 6 & Coronil, El & 171903 & 181779 & 211692 & 226608 & 246477 & 253591 & 262614 & 152,77 \\
\hline 6 & Aguadulce & 171172 & 181004 & 193941 & 197242 & 237938 & 248583 & 261098 & 152,54 \\
\hline 1 & Castillo Guardas & 212090 & 224377 & 248911 & 270608 & 309346 & 316012 & 322677 & 152,14 \\
\hline 5 & Huevar & 178910 & 189207 & 201113 & 214878 & 230793 & 249193 & 270468 & 151,18 \\
\hline 6 & Corrales, Los & 143994 & 152196 & 170169 & 184406 & 194511 & 201180 & 217486 & 151,04 \\
\hline 1 & Gerena & 184379 & 195545 & 218475 & 235070 & 238343 & 247994 & 277494 & 150,50 \\
\hline
\end{tabular}


TABLA NÚM. 2.-CLASIFICACIÓN POR ORDEN DECRECIENTE DEL ÍNDICE DE AUMENTO DE LA RENTA PERSONAL

RENTA PER CÁPITA (PTAS DE 1980)

\begin{tabular}{|c|c|c|c|c|c|c|c|c|c|}
\hline $\begin{array}{l}\text { NUM. } \\
\text { COMARC }\end{array}$ & MUNICIPIOS & 1977 & 1980 & 1982 & 1984 & 1986 & 1987 & 1988 & $\begin{array}{l}\text { ÍNDICE } \\
\text { R'CRMTO. } \\
1988.87\end{array}$ \\
\hline 5 & Villanueva Ariscal & 251667 & 266330 & 300871 & 332645 & 344074 & 355503 & 378009 & 150,20 \\
\hline 2 & Algaba, La & 181791 & 202283 & 220517 & 226560 & 246099 & 247077 & 271628 & 149,42 \\
\hline 5 & Bollullos Mitación & 193425 & 204592 & 217311 & 231795 & 248290 & 267075 & 288469 & 149,14 \\
\hline 3 & Paradas & 168886 & 178581 & 197401 & 210179 & 236714 & 232952 & 251801 & 149,10 \\
\hline 1 & Pedroso, El & 180263 & 190641 & 210198 & 216689 & 230208 & 240148 & 267710 & 148,51 \\
\hline 3 & Arahal, El & 173937 & 183935 & 206322 & 225688 & 238836 & 244077 & 257769 & 148,20 \\
\hline 2 & Alcalá del Río & 200211 & 211786 & 227937 & 252494 & 260118 & 257656 & 296362 & 148,02 \\
\hline 3 & Lebrija & 177832 & 188063 & 209839 & 228829 & 239464 & 244758 & 263049 & 147,92 \\
\hline 3 & Cabezas San Juan & 184108 & 194717 & 224470 & 237981 & 254081 & 259779 & 272201 & 147,85 \\
\hline 4 & Puebla del Río & 224474 & 237505 & 266693 & 285505 & 323066 & 323066 & 331675 & 147,76 \\
\hline 6 & Martín de la Jara & 146962 & 155343 & 171912 & 193482 & 191068 & 202099 & 215520 & 146.65 \\
\hline & Coria del Río & 219423 & 232151 & 270316 & 293661 & 317375 & 316881 & 321678 & 146,60 \\
\hline & Media provincial & 196166 & 206885 & 228918 & 244632 & 260767 & 268796 & 287414 & 146,52 \\
\hline 2 & Tocina & 193992 & 205194 & 264566 & 253950 & 273953 & 278982 & 283677 & 146,23 \\
\hline 1 & Puebla Infantes & 157551 & 166566 & 189402 & 200644 & 211282 & 216582 & 229579 & 145,72 \\
\hline 4 & Camas & 230023 & 243386 & 253895 & 288032 & 309027 & 3118112 & 334542 & 145,44 \\
\hline 3 & Palacios, Los & 186516 & 197270 & 222108 & 232199 & 252022 & 259562 & 270515 & 145,04 \\
\hline 5 & Palomares del Río & 273748 & 289735 & 307312 & 326637 & 347884 & 371244 & 396928 & 145,00 \\
\hline 1 & Cantillana & 199533 & 211068 & 229462 & 244759 & 268593 & 268567 & 289220 & 144,95 \\
\hline 2 & Peñaflor & 176184 & 186318 & 206504 & 220059 & 225916 & 226736 & 255036 & 144,76 \\
\hline 6 & Marinaleda & 152135 & 160826 & 178024 & 187661 & 201419 & 28735 & 219539 & 144,31 \\
\hline 6 & Montellano & 161647 & 171409 & 189627 & 205047 & 203556 & 218150 & 233041 & 144,17 \\
\hline 1 & Guadalcanal & 190507 & 201500 & 216752 & 225459 & 224387 & 232994 & 274383 & 144,03 \\
\hline 3 & Lantejuela, La & 179466 & 189797 & 222602 & 235262 & 239464 & 244758 & 263049 & 147,92 \\
\hline 6 & Badalatosa & 159890 & 169046 & 179780 & 193751 & 201007 & 217741 & 228751 & 143,07 \\
\hline 6 & Morón Frontera & 202282 & 213982 & 240768 & 252097 & 263427 & 272057 & 289285 & 143,01 \\
\hline 5 & Salteras & 213947 & 226346 & 248973 & 256705 & 277285 & 291402 & 305913 & 142,99 \\
\hline 4 & Gelves & 227645 & 240865 & 270904 & 277960 & 283219 & 288413 & 324769 & 142,66 \\
\hline 6 & Casariche & 166259 & 175797 & 195045 & 210255 & 209419 & 215542 & 237101 & 142,61 \\
\hline 5 & Pilas & 210870 & 223084 & 230878 & 254443 & 263794 & 274445 & 300042 & 142,29 \\
\hline 4 & Alcalá de Guadaira & 254845 & 269698 & 296347 & 305877 & 328370 & 333775 & 362233 & 142,14 \\
\hline 3 & Fuentes Andalucía & 176736 & 186902 & 176785 & 187836 & 219316 & 226374 & 250942 & 141,99 \\
\hline 4 & Santiponce & 213223 & 225579 & 255595 & 274743 & 270850 & 273676 & 302342 & 141,80 \\
\hline 5 & Villamanrique Cond. & 176830 & 187002 & 179670 & 187445 & 251163 & 256481 & 250725 & 141,79 \\
\hline 5 & Espartinas & 288242 & 305099 & 355575 & 358724 & 404198 & 407235 & 408493 & 141,72 \\
\hline 5 & Bormujos & 210910 & 223126 & 242360 & 262604 & 270480 & 278771 & 298585 & 141,57 \\
\hline 5 & Mairena del Aljarafe & 270589 & 286386 & 303767 & 322889 & 343929 & 367078 & 382521 & 141,37 \\
\hline
\end{tabular}


TABLA NÚM. 2.-CLASIFICACIÓN POR ORDEN DECRECIENTE DEL ÍNDICE DE AUMENTO DE LA RENTA PERSONAL

RENTA PER CÁPITA (PTAS DE 1980)

\begin{tabular}{|c|c|c|c|c|c|c|c|c|c|}
\hline $\begin{array}{l}\text { NUM. } \\
\text { COMARC }\end{array}$ & MUNCIPIOS & 1977 & 1980 & 1982 & 1984 & 1986 & 1987 & 1988 & $\begin{array}{l}\text { ÁDICR } \\
\text { RNCRMTO } \\
1988.87\end{array}$ \\
\hline 6 & Saucejo,El & 148317 & 156779 & 170714 & 180998 & 188793 & 200608 & 209648 & 141,35 \\
\hline 2 & Lora del Rio & 189649 & 200590 & 215174 & 225956 & 239292 & 246337 & 267915 & 141,27 \\
\hline 4 & S.Juan Aznalfarache & 275494 & 291586 & 320543 & 338542 & 406216 & 396417 & 386944 & 140,45 \\
\hline 3 & Campana, $\mathrm{La}$ & 197804 & 209235 & 220061 & 232104 & 245501 & 260406 & 276987 & 140,03 \\
\hline 6 & Estepa & 197657 & 209079 & 222274 & 244606 & 260479 & 265047 & 276147 & 139,71 \\
\hline 5 & Olivares & 201341 & 212983 & 239714 & 255099 & 258551 & 266093 & 280798 & 139,46 \\
\hline 2 & Rinconada,La & 216543 & 229098 & 251129 & 263178 & 275411 & 289890 & 301193 & 139,09 \\
\hline 6 & Roda de Andalucia & 187761 & 198589 & 214169 & 228231 & 232242 & 237999 & 260230 & 138,60 \\
\hline 5 & Gines & 310281 & 328460 & 387276 & 399440 & 411604 & 419964 & 428323 & 138,04 \\
\hline 5 & Aznalcazar & 187137 & 197927 & 219699 & 235682 & 242939 & 251366 & 258108 & 137,92 \\
\hline 1 & Burguillos & 181343 & 191786 & 212995 & 213166 & 239548 & 242240 & 249760 & 137,73 \\
\hline 6 & Rubio,El & 174587 & 184625 & 197642 & 209069 & 222705 & 233385 & 240252 & 137,61 \\
\hline 6 & Algamitas & 157919 & 166957 & 240234 & 225354 & 207247 & 211605 & 216164 & 136,88 \\
\hline 2 & Villaverde del Rio & 194444 & 205673 & 217224 & 228254 & 253398 & 258887 & 265601 & 136,60 \\
\hline 6 & Pedrera & 170620 & 180420 & 201441 & 209605 & 226670 & 237022 & 232891 & 136,50 \\
\hline 1 & Alanis & 164547 & 173981 & 184246 & 195206 & 197681 & 203882 & 223658 & 135,92 \\
\hline 5 & Carrión Céspedes & 182121 & 192610 & 214470 & 225956 & 226662 & 237343 & 247087 & 135,67 \\
\hline 5 & Castilleja de Guzmán & 205877 & 217792 & 185858 & 253375 & 267406 & 272258 & 277261 & 134,67 \\
\hline 4 & Sevilla & 326174 & 345307 & 334699 & 365348 & 408018 & 408540 & 432501 & 132,60 \\
\hline 5 & Sanlucar la Mayor & 262814 & 278145 & 313406 & 321310 & 331676 & 338958 & 346483 & 131,84 \\
\hline 2 & Alcolea del Rio & 191481 & 202441 & 211311 & 219024 & 244633 & 246924 & 245875 & 128,41 \\
\hline 4 & Dos Hermanas & 229931 & 243290 & 253023 & 273824 & 274767 & 281952 & 294972 & 128,29 \\
\hline 1 & Real de la Jara & 155839 & 164752 & 159476 & 164810 & 193724 & 196443 & 199254 & 127,86 \\
\hline \multirow[t]{2}{*}{5} & Albaida Aljarafe & 193513 & 204686 & 217907 & 234075 & 227186 & 240283 & 247049 & 127,67 \\
\hline & Media nacional & 319012 & 349265 & 349743 & 362058 & 359444 & 378291 & 399894 & 125,35 \\
\hline 1 & Almadén de la Plata & 166997 & 176579 & 222223 & 233132 & 192049 & 196268 & 200713 & 120,19 \\
\hline 5 & Castilleja cuesta & 318368 & 337032 & 358985 & 351907 & 368097 & 366766 & 376109 & 118,14 \\
\hline 1 & San Nicolfs Puerto & 184713 & 188966 & 193472 & 198244 & 203300 & 208656 & 214329 & 116,03 \\
\hline 1 & Garrobo,El & 262577 & 220177 & 259766 & 267688 & 276038 & 284838 & 294114 & 112,01 \\
\hline \multirow[t]{2}{*}{1} & Aznalcollar & 186475 & 197226 & 224965 & 235759 & 184671 & 187797 & 191059 & 102,46 \\
\hline & $\%$ md. prov. s/nac. & 71,02 & 89,58 & 86,60 & 88,07 & 66,90 & 65,93 & 64,96 & \\
\hline
\end{tabular}


TABLA NÚM. 3-RENTA MUNICIPAL POR COMARCAS (MUNICIPIOS ORDENADOS POR INDICES DECRECIENYES)

RENTA PER CÁPITA (PTAS DE 1980)

\begin{tabular}{|c|c|c|c|c|c|c|c|c|c|}
\hline $\begin{array}{l}\text { NÚM. } \\
\text { COMARC }\end{array}$ & MUNICIPIOS & 1977 & 1980 & 1982 & 1984 & 1986 & 1987 & 1988 & $\begin{array}{l}\text { ÍNDICE } \\
\text { INCRMTO } \\
1988.87\end{array}$ \\
\hline & SIERRA NORTE & & & & & & & & \\
\hline 1 & Constatina & 167143 & 176734 & 223268 & 244874 & 264336 & 262872 & 278203 & 166,45 \\
\hline 1 & Villan,Rio y Minas & 178839 & 189131 & 216954 & 224147 & 275579 & 281213 & 287042 & 160,50 \\
\hline 1 & Fastiblanco Aпroyos & 181427 & 191876 & 220785 & 247516 & 256763 & 245941 & 291066 & 160,43 \\
\hline 1 & Madroño,El & 171298 & 181138 & 208353 & 222663 & 243123 & 257674 & 272127 & 158,86 \\
\hline 1 & Navas Concepción & 158770 & 167859 & 187964 & 197929 & 192454 & 200398 & 247720 & 156,02 \\
\hline 1 & Ronquillo, El & 193574 & 204750 & 214916 & 221667 & 250945 & 257674 & 298680 & 154,30 \\
\hline 1 & Cazalla de la Sierra & 170233 & 180009 & 199245 & 215502 & 231459 & 240483 & 261347 & 153,52 \\
\hline 1 & Castillo Guardas & 212090 & 224377 & 248911 & 270608 & 309346 & 316012 & 322677 & 152,14 \\
\hline 1 & Gerena & 184379 & 195545 & 218475 & 235070 & 238343 & 247994 & 277494 & 150,50 \\
\hline 1 & Pedroso,El & 180263 & 190641 & 210198 & 216689 & 230208 & 240148 & 267710 & 148,51 \\
\hline 1 & Puebla Infantes & 157551 & 166566 & 189402 & 200644 & 211282 & 216582 & 229579 & 145,72 \\
\hline 1 & Cantillana & 199533 & 211068 & 229462 & 244759 & 268593 & 268567 & 289220 & 144,95 \\
\hline 1 & Guadalcanal & 190507 & 201500 & 216752 & 225459 & 224387 & 232994 & 274383 & 144,03 \\
\hline 1 & Burguillos & 181343 & 191786 & 212995 & 213166 & 239548 & 242240 & 249760 & 137,73 \\
\hline 1 & Alanis & 164547 & 173981 & 184246 & 195206 & 197681 & 203882 & 223658 & 135,92 \\
\hline 1 & Real de la Jara & 155839 & 164752 & 159476 & 164810 & 193724 & 196443 & 199254 & 127,86 \\
\hline 1 & Almadén de la Plata & 166997 & 176579 & 222223 & 233132 & 192049 & 196268 & 200713 & 120,19 \\
\hline 1 & San Nicolás Puerto & 184713 & 188966 & 193472 & 198244 & 203300 & 208656 & 214329 & 116,03 \\
\hline 1 & Garrobo, El & 262577 & 220177 & 259766 & 267688 & 276038 & 284838 & 294114 & 112,01 \\
\hline & Aznalcollar & 186475 & 197226 & 224965 & 235759 & 184671 & 187797 & 191059 & 102,46 \\
\hline & Media Comarcal & 179405 & 189666 & 214278 & 227563 & 239177 & 242988 & 262125 & 146,11 \\
\hline & Media Provincial & 256918 & 273683 & 282594 & 305636 & 332378 & 336493 & 358168 & 139,41 \\
\hline & Media Nacional & 310012 & 349265 & 349743 & 362058 & 359444 & 378291 & 399894 & 128,99 \\
\hline & Fomed.comre s/md,prov & 69,83 & 69,30 & 75,83 & 74,46 & 71,96 & 72,21 & 73,18 & \\
\hline & $\begin{array}{l}\text { Jomed.comre simd. nac } \\
\text { VALLEGUADALQUIVIR }\end{array}$ & 57,87 & 54,30 & 61,27 & 62,85 & 66,54 & 64,23 & 65,55 & \\
\hline 2 & Guillena & 175836 & 185949 & 208421 & 225879 & 242677 & 247545 & 274081 & 155,87 \\
\hline 2 & Brenes & 198498 & 209971 & 234622 & 249699 & 254511 & 260812 & 303355 & 152,83 \\
\hline 2 & Algaba, La & 181791 & 202283 & 220517 & 226560 & 246099 & 247077 & 271628 & 149,42 \\
\hline 2 & Alcalá del Rio & 200211 & 211786 & 227937 & 252494 & 260118 & 257656 & 296362 & 148,02 \\
\hline 2 & Tocina & 193992 & 205194 & 264566 & 253950 & 273953 & 278982 & 283677 & 146,23 \\
\hline 2 & Peñaflor & 176184 & 186318 & 206504 & 220059 & 225916 & 226736 & 255036 & 144,76 \\
\hline 2 & Lora del Rio & 189649 & 200590 & 215174 & 225956 & 239292 & 246337 & 267915 & 141,27 \\
\hline 2 & Rinconada, La & 216543 & 229098 & 251129 & 263178 & 275411 & 289899 & 301193 & 139,09 \\
\hline 2 & Villaverde del Rio & 194444 & 205673 & 217224 & 228254 & 253398 & 258887 & 265601 & 136,60 \\
\hline 2 & Alcolea del Rio & 191481 & 202441 & 211311 & 219024 & 244633 & 246924 & 245875 & 128,41 \\
\hline
\end{tabular}


TABLA NUM.3. RENTA MUNICIPAL POR COMARCAS (MUNICIPIOS ORDENADOS POR INDICES DECRECIENTES)

RENTA PER CÁPITA (PTAS DE 1980)

\begin{tabular}{|c|c|c|c|c|c|c|c|c|c|}
\hline $\begin{array}{c}\text { NÚM, } \\
\text { COMAARC }\end{array}$ & MUNICIPIOS & 1977 & 1980 & 1982 & 1984 & 1986 & 1987 & 1988 & $\begin{array}{l}\text { 'SDICE } \\
\text { R'CRMTO } \\
1988.87\end{array}$ \\
\hline & Media Comarcal & 191548 & 207375 & 229040 & 240015 & 254460 & 260504 & 281518 & 146,97 \\
\hline & Media Provincial & 256918 & 273683 & 282594 & 305636 & 332378 & 336493 & 358168 & 139,41 \\
\hline & Media Nacional & 310012 & 349265 & 349743 & 362058 & 359444 & 378291 & 399894 & 128,99 \\
\hline & $\%$ med. comre simd.pror & 74,56 & 75,77 & 81,05 & 78,53 & 76,56 & 77,42 & 78,60 & \\
\hline & $\begin{array}{l}\% \text { med. comre simd nac. } \\
\text { CAMPNंA-MARISMAS }\end{array}$ & 61,79 & 39,37 & 65,49 & 66,29 & 70,79 & 68,86 & 70,40 & \\
\hline 3 & Mairena del Alcor & 221051 & 233876 & 277298 & 316178 & 325682 & 333672 & 430136 & 194,59 \\
\hline 3 & Carmona & 217944 & 230584 & 253901 & 304537 & 322134 & 328324 & 378168 & 173,52 \\
\hline 3 & Marchena & 193998 & 205190 & 264384 & 284547 & 300174 & 309261 & 335432 & 172,90 \\
\hline 3 & Luisiana, La & 149390 & 157916 & 196533 & 208494 & 223194 & 229064 & 246660 & 165,11 \\
\hline 3 & Viso del Alcor, El & 188213 & 199068 & 233853 & 251498 & 255528 & 266576 & 306322 & 162,75 \\
\hline 3 & Ecija & 197982 & 209423 & 240715 & 261264 & 291667 & 296717 & 316176 & 159,70 \\
\hline 3 & Osuna & 178459 & 188729 & 230692 & 245372 & 274439 & 277320 & 281160 & 157,55 \\
\hline 3 & Puebla de Cazalla & 179033 & 179882 & 208276 & 228522 & 241080 & 246721 & 279453 & 156,09 \\
\hline 3 & Molares, Los & 153381 & 162146 & 187604 & 197548 & 208818 & 221589 & 236063 & 153,91 \\
\hline 3 & Utrera & 200616 & 212215 & 234217 & 253153 & 268992 & 276785 & 306943 & 153,00 \\
\hline 3 & Paradas & 168886 & 178581 & 197401 & 210179 & 236714 & 232952 & 251801 & 149,10 \\
\hline 3 & Arahal, El & 173937 & 183935 & 206322 & 225688 & 238836 & 244077 & 257769 & 148,20 \\
\hline 3 & Lebrija & 177832 & 188063 & 209839 & 228829 & 239464 & 244758 & 263049 & 147,92 \\
\hline 3 & Cabezas San Juan & 184108 & 194717 & 224470 & 237981 & 254081 & 259779 & 272201 & 147,85 \\
\hline 3 & Palacios,Los & 186516 & 197270 & 222108 & 232199 & 252022 & 259562 & 270515 & 145,04 \\
\hline 3 & Lantejuela, La & 179466 & 189797 & 222602 & 235262 & 236353 & 238817 & 257859 & 143,68 \\
\hline 3 & Fuentes Andalucía & 176736 & 186902 & 176785 & 187836 & 219316 & 226374 & 250942 & 141,99 \\
\hline \multirow[t]{6}{*}{3} & Campana, La & 197804 & 209235 & 220061 & 232104 & 245501 & 260406 & 276987 & 140,03 \\
\hline & Media Comarcal & 190151 & 201135 & 229494 & 250083 & 267807 & 274190 & 300399 & 157,98 \\
\hline & Media Provincial & 256918 & 273683 & 282594 & 305636 & 332378 & 336493 & 358168 & 139,41 \\
\hline & Media Nacional & 310012 & 349265 & 349743 & 362058 & 359444 & 378291 & 399894 & 128,99 \\
\hline & to med. comse simd.prov. & 74,01 & 73,49 & 81,21 & 81,82 & 80,57 & 81,48 & 83,87 & \\
\hline & $\begin{array}{l}\text { \% med.comrc s/md.nac. } \\
\text { ZONA LFFLUESCCAPPTAL }\end{array}$ & 61,34 & 57,59 & 65,62 & 69,07 & 74,51 & 72,48 & 75,12 & \\
\hline 4 & Puebla del io & 224474 & 237505 & 266693 & 285505 & 323066 & 324197 & 331675 & 147,76 \\
\hline 4 & Coria del Rio & 219423 & 232151 & 270316 & 293661 & 317375 & 316881 & 321678 & 146,60 \\
\hline 4 & Camas & 230023 & 243386 & 253895 & 288032 & 309027 & 311812 & 334542 & 145,44 \\
\hline 4 & Gelves & 227645 & 240865 & 270904 & 277960 & 283219 & 288413 & 324769 & 142,66 \\
\hline 4 & Alcalá de Guadaira & 254845 & 269698 & 296347 & 305877 & 328370 & 333775 & 362233 & 142,14 \\
\hline 4 & Santiponce & 213223 & 225579 & 255595 & 274743 & 270850 & 273676 & 302342 & 141,80 \\
\hline 4 & S.Juan Aznalfarache & 275494 & 291586 & 320543 & 338542 & 406216 & 396417 & 386944 & 140,45 \\
\hline
\end{tabular}


TABLA NÚM. 3-RENTA MUNICIPAL POR COMARCAS (MUNICIPIOS ORDENADOS POR INDICES DECRECIENYES)

RENTA PER CÁPITA (PTAS DE 1980)

\begin{tabular}{|c|c|c|c|c|c|c|c|c|c|}
\hline $\begin{array}{l}\text { NÚM. } \\
\text { COMARC }\end{array}$ & MUNICIPIOS & 1977 & 1980 & 1982 & 1984 & 1986 & 1987 & 1988 & $\begin{array}{l}\text { ÍNDICE } \\
\text { INCRMT0 } \\
1988.87\end{array}$ \\
\hline 4 & Sevilla & 326174 & 345307 & 334699 & 365348 & 408018 & 408540 & 432501 & 132,60 \\
\hline \multirow[t]{6}{*}{4} & Dos Hermanas & 229931 & 243290 & 253023 & 273824 & 274767 & 281952 & 294972 & 128,29 \\
\hline & Media Comarcal & 306416 & 323994 & 320742 & 348771 & 384819 & 385930 & 408607 & 133,35 \\
\hline & Media Provincial & 256918 & 273683 & 282594 & 305636 & 332378 & 336493 & 358168 & 139,41 \\
\hline & Media Nacional & 310012 & 349265 & 349743 & 362058 & 359444 & 378291 & 399894 & 128,99 \\
\hline & Fomed. comre simd. prov & 119,27 & 118,38 & 113,50 & 114,11 & 115,78 & 114,69 & 114,08 & \\
\hline & $\begin{array}{c}\text { \%omed.comre simd. nac. } \\
\text { ALJARAFE, El }\end{array}$ & 98,84 & 92,76 & 91,71 & 96,33 & 107,06 & 102,02 & 102,18 & \\
\hline 5 & Benacazón & 179761 & 190109 & 212126 & 233714 & 260004 & 292019 & 331006 & 184,14 \\
\hline 5 & Almensilla & 190118 & 201087 & 182617 & 257204 & 274714 & 275974 & 331683 & 174,46 \\
\hline 5 & Valencina Concep. & 302584 & 320302 & 338853 & 363549 & 438511 & 468571 & 495429 & 163,73 \\
\hline 5 & Castilleja del Campo & 202716 & 214441 & 222872 & 246597 & 262912 & 290277 & 322637 & 159,16 \\
\hline 5 & Umbrete & 200341 & 211924 & 253401 & 263715 & 281178 & 291831 & 317752 & 158,61 \\
\hline 5 & Tomares & 266969 & 282550 & 329590 & 348614 & 351276 & 381155 & 411033 & 153,96 \\
\hline 5 & Huevar & 178910 & 189207 & 201113 & 214878 & 230793 & 249193 & 270468 & 151,18 \\
\hline 5 & Villanueva Ariscal & 251667 & 266330 & 300871 & 332645 & 344074 & 355503 & 378009 & 150,20 \\
\hline 5 & Bollullos Mitación & 193425 & 204592 & 217311 & 231795 & 248290 & 267075 & 288469 & 149,14 \\
\hline 5 & Palomares del Rio & 273748 & 289735 & 307312 & 326637 & 347884 & 371244 & 396928 & 145,00 \\
\hline 5 & Salteras & 213947 & 226346 & 248973 & 256705 & 277285 & 291402 & 305913 & 142,99 \\
\hline 5 & Pilas & 210870 & 223084 & 230878 & 254443 & 263794 & 274445 & 300042 & 142,29 \\
\hline 5 & Villamanrique Cond. & 176830 & 187002 & 179670 & 187445 & 251163 & 256481 & 250725 & 141,79 \\
\hline 5 & Espartinas & 288242 & 305099 & 355575 & 358724 & 404198 & 407235 & 408493 & 141,72 \\
\hline 5 & Bormujos & 210910 & 223126 & 242360 & 262604 & 270480 & 278771 & 298585 & 141,57 \\
\hline 5 & Mairena del Aljarafe & 270589 & 286386 & 303767 & 322889 & 343929 & 367078 & 382521 & 141,37 \\
\hline .5 & Olivares & 201341 & 212983 & 239714 & 255099 & 258551 & 266093 & 280798 & 139,46 \\
\hline 5 & Gines & 310281 & 328460 & 387276 & 399440 & 411604 & 419964 & 428323 & 138,04 \\
\hline 5 & Aznalcazar & 187137 & 197927 & 219699 & 235682 & 242939 & 251366 & 258108 & 137,92 \\
\hline 5 & Carrión Céspedes & 182121 & 192610 & 214470 & 225956 & 226662 & 237343 & 247087 & 135,67 \\
\hline 5 & Castilleja de Guzmán & 205877 & 217792 & 185858 & 253375 & 267406 & 272258 & 277261 & 134,67 \\
\hline 5 & Sanlucar la Mayor & 262814 & 278145 & 313306 & 321310 & 331676 & 338958 & 346483 & 131,84 \\
\hline 5 & Albaida Aljarafe & 193513 & 204686 & 217907 & 234075 & 227186 & 240283 & 247049 & 127,67 \\
\hline \multirow[t]{6}{*}{5} & Castilleja Cuesta & 318368 & 337032 & 358985 & 351907 & 368097 & 366766 & 376109 & 118,14 \\
\hline & Media Comarcal & 237726 & 256154 & 280193 & 295227 & 315191 & 330546 & 350056 & 147,25 \\
\hline & Media Provincial & 256918 & 273683 & 282594 & 305636 & 332378 & 336493 & 358168 & 139,41 \\
\hline & Media Nacional & 310012 & 349265 & 349743 & 362058 & 359444 & 378291 & 399894 & 128,99 \\
\hline & Tomed.cmre s/md.prov & 92,53 & 93,60 & 99,15 & 96,59 & 94,83 & 98,23 & 97,74 & \\
\hline & Fomed.comrc s/md.nac. & 76,68 & 73,34 & 80,11 & 81,54 & 87,69 & 87,38 & 87,34 & \\
\hline
\end{tabular}


TABLA NUMM. RENTA MUNICIPAL POR COMARCAS (MUNICIPIOS ORDENADOS POR LNDICES DECRECIENTES)

RENTA PER CÁPITA (PTAS DE 1980)

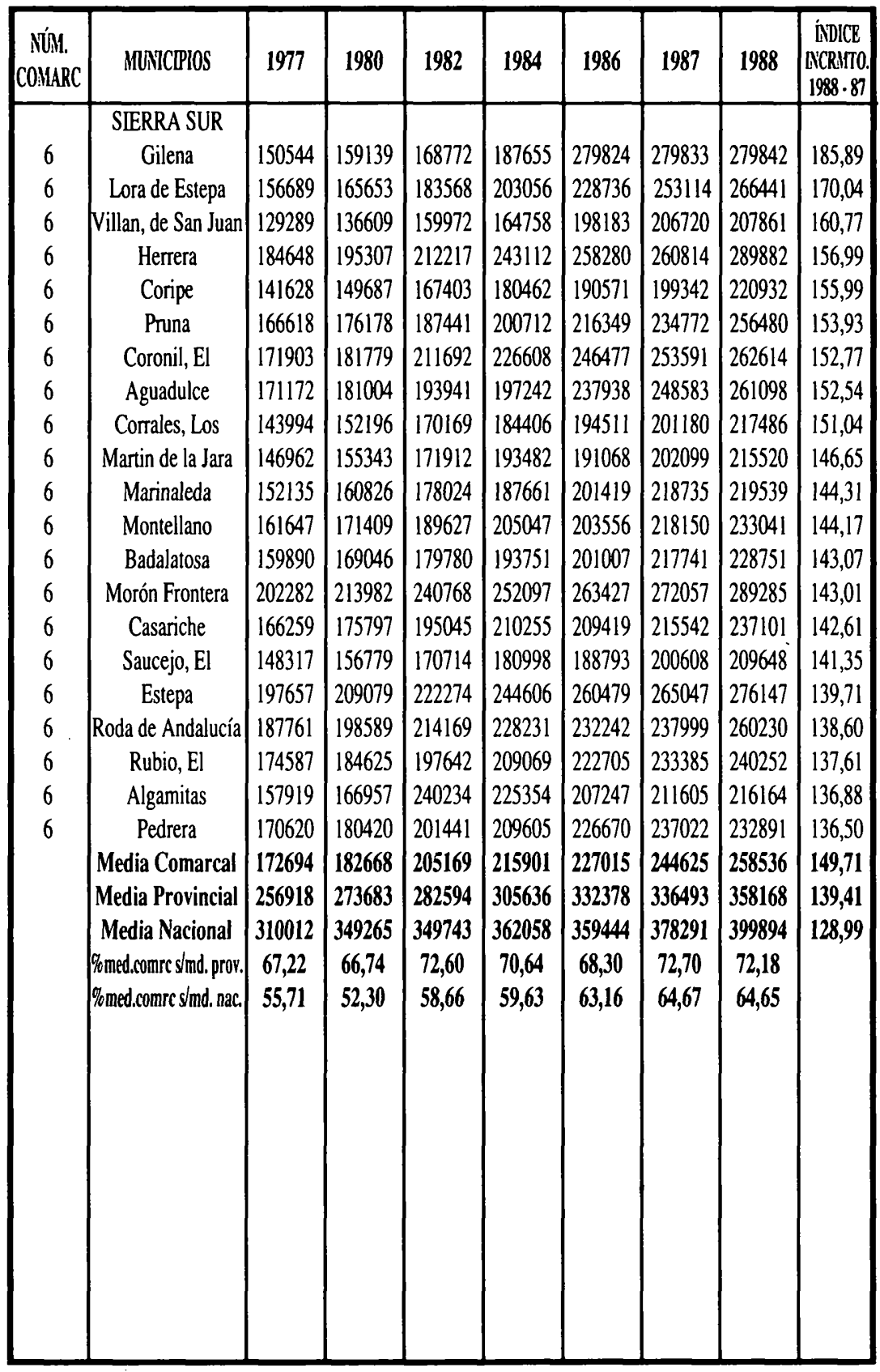




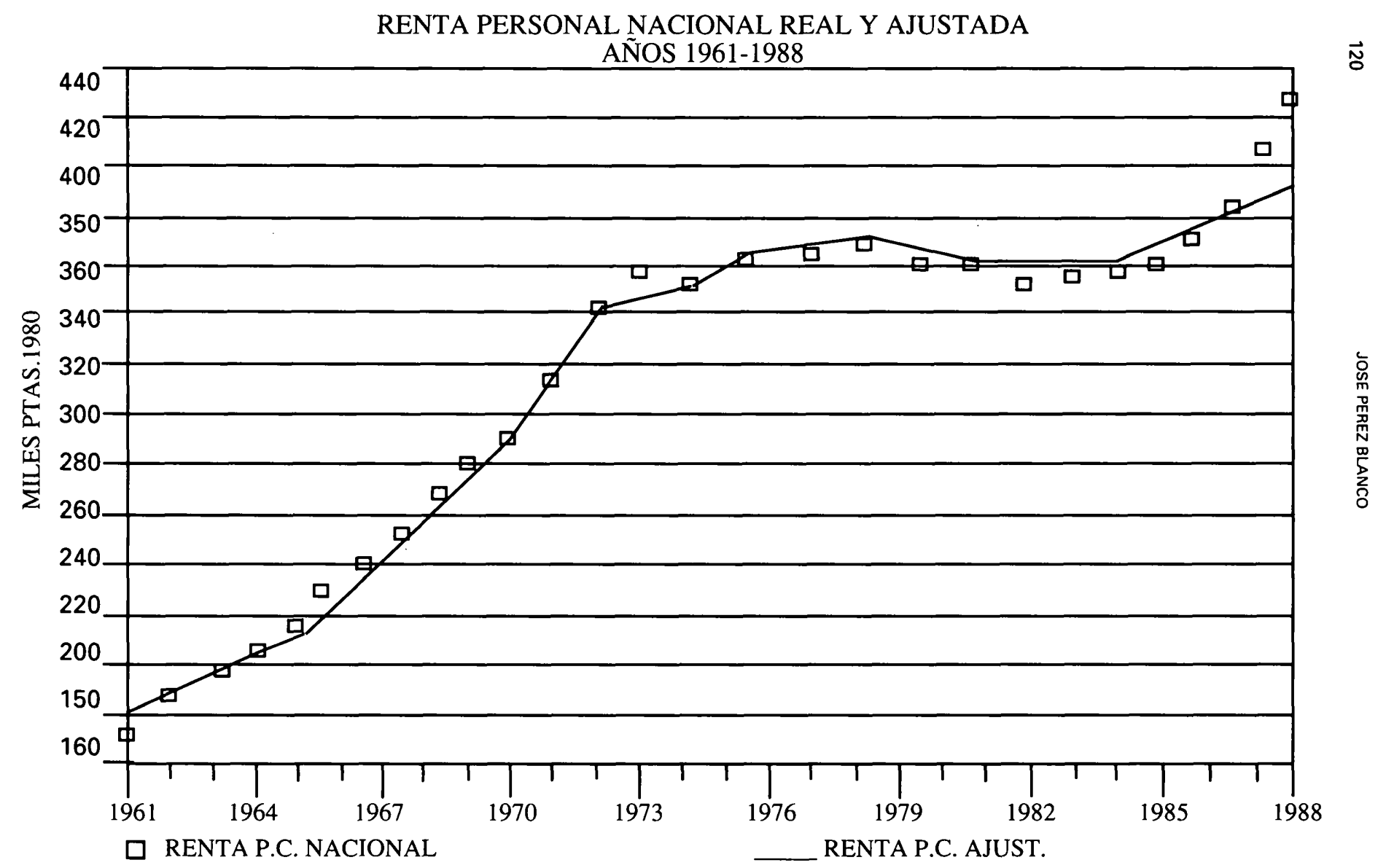

